\title{
Risk factors for death among tuberculosis cases: analysis of European surveillance data
}

\author{
N. Lefebvre ${ }^{\star, *}$ and D. Falzon*
}

ABSTRACT: The proportion of tuberculosis (TB) patients in the European Union (EU) who die remains high ( $8 \%$ overall). The aim of the present study was to quantify the risk of dying associated with demographic and clinical factors.

Case-based data on 39,566 TB patients notified by 15 EU countries during 2002-2004 were analysed using logistic regression.

It was observed that advancing age and resistance to isoniazid and rifampicin were the strongest determinants of death, while male sex, European origin, pulmonary site of disease and previous anti-TB treatment were weaker predictors. Risk varied between reporting countries, presumably reflecting differences in patient profiles, reporting practices and programme effectiveness.

In conclusion, earlier suspicion, diagnosis and treatment may reduce deaths, particularly among the elderly. Special attention is needed to avert the development and transmission of multidrug-resistant tuberculosis.

KEYWORDS: Death, epidemiology, European Union, risk factors, tuberculosis

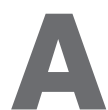
lthough tuberculosis (TB) mortality has reached very low levels in the developed world, the proportion of pulmonary TB cases who die in most countries of the European Union (EU) is one of the highest in the world [1]. The burden of TB deaths in Europe is higher than that of other key infectious diseases [2]. In certain classes of TB patients, the risk of dying could be reduced through improved care [3,4]. Studying the determinants of death in TB patients could help both the public health worker and the clinician alike to identify vulnerable individuals and important factors warranting more targeted action. The present authors used surveillance data on recent $\mathrm{TB}$ notifications from 15 EU countries to quantify the risk of dying.

\section{MATERIALS AND METHODS}

European countries annually report national TB surveillance data to EuroTB, a network funded by the European Commission [5]. Of $27 \mathrm{EU}$ countries, 20 countries now report outcomes, including death, as part of an anonymised individual dataset. Submission of data is based on consensus recommendations and the methodology used has been described elsewhere [6]. A case was classified as "died" if the first outcome observed within 12 months of case detection was death from any cause. In the present study, a previous history of TB was defined as a past episode of curative combination therapy with
anti-TB drugs for $\geqslant 1$ month. Geographical origin was determined by the patient's place of birth or citizenship in three countries (Austria, Belgium and the Netherlands). Cases with both pulmonary and extrapulmonary TB were included under "pulmonary". Multidrug resistance (MDR) was defined as resistance to both isoniazid and rifampicin at initial drug susceptibility testing.

Deaths observed among TB patients were compared with those expected from national mortality statistics. Data for total deaths were obtained from Eurostat [7] and population statistics from the United Nations Populations Division [8].

Bivariate and multivariable analyses were restricted to culture-positive patients with complete data for plausible determinants of death available on the European dataset (sex, age, geographical origin, site of disease, previous history of $\mathrm{TB}$, and drug susceptibility testing results). To enhance comparability, countries were only included if $\geqslant 50 \%$ of their reported cases had been confirmed by culture and if initial drug susceptibility testing results were available for $\geqslant 80 \%$ of culture-positive cases. Countries reporting $<30$ deaths $\cdot \mathrm{yr}^{-1}$ were excluded. As the present study was carried out using routinely collected, anonymous surveillance data, ethical clearance was not deemed necessary.

The "null hypothesis" of no association between death and putative explanatory variables was
AFFILIATIONS

*EuroTB, Dept of Infectious Diseases, Institut de Veille Sanitaire, SaintMaurice, and

\#Dept of Infectious Diseases and Tropical Medicine, University Hospital, Strasbourg, France.

CORRESPONDENCE

D. Falzon

EuroTB

Dept of Infectious Diseases

Institut de Veille Sanitaire

12 rue du Val d'Osne

94415 Saint-Maurice Cedex

France

Fax: 33141796802

E-mail: d.falzon@invs.sante.fr

Received:

October 052007

Accepted after revision:

January 092008

SUPPORT STATEMENT

The EuroTB network is funded by the European Commission's Health and Consumer Protection Directorate General (DG SANCO) and by the Institut de Veille Sanitaire, SaintMaurice, France.

STATEMENT OF INTEREST None declared. 
tested using data pooled from all countries for all 3 yrs. Bivariate analysis was employed to assess the association between death and year of report, sex, age group, region of origin, site of disease, previous history of TB, MDR and country of report, using the Chi-squared test to assess statistical significance (a p-value $<0.05$ was considered significant). Logistic regression was used to identify variables independently associated with risk of death. MDR was stratified by previous history of TB with a term of interaction to adjust for effect modification (no MDR; primary MDR, i.e. MDR without previous history of TB; and secondary MDR, i.e. with previous history). Results of bivariate analysis and logistic regression were reported as crude and adjusted odds ratio (OR), respectively, with $95 \%$ confidence intervals (CI).

\section{RESULTS}

In total, 15 countries (Austria, Belgium, the Czech Republic, Denmark, Estonia, Germany, Ireland, Latvia, Lithuania, the Netherlands, Portugal, Slovakia, Slovenia, Sweden and the UK) satisfied the selection criteria for the study. Deaths among TB cases reported in 2003 were compared with those expected in 13 countries with available national mortality data (table 1). While the proportion of subjects dying increased progressively with age among TB cases, the ratio of observed to expected deaths was highest in young adults.

In the period 2002-2004, 82,314 TB cases in total were notified by the 15 countries, of whom 52,479 (64\%) were culturepositive and 46,151 (56\%) had drug susceptibility testing results available. A total of $39,566(48 \%)$ cases with complete data for the variables of interest were retained for the rest of the analysis (table 2). Three countries reported half of the cases: Germany (24\%), the UK (15\%) and Portugal (11\%). Onefifth of cases were reported by the three Baltic States (Estonia, Latvia and Lithuania).

TB cases were predominantly male $(65 \%)$ and the median age was 43 yrs, with $2 \%$ aged $<15$ yrs and $24 \%$ aged $\geqslant 60$ yrs. Most cases were European (68\%), 12\% were African and $6 \%$ originated from the Indian subcontinent (definitions are given in table 2). Most cases had pulmonary disease (84\%) and no previous history of TB (86\%). Only $5 \%$ of patients had MDR, but this proportion was higher in the Baltic States than in the other 12 countries (19.6 versus $1.6 \%$, respectively; $\mathrm{p}<.001$ ). Patients with a previous history of TB were more likely to have MDR (20.2 versus $2.8 \%$; $<0.001)$. Overall, 3,085 (7.8\%) cases died (ranging 5-12\% between countries), 29,184 (73.8\%) cases completed treatment (country range 56-86\%) and 7,297 (18.5\%; country range $2-37 \%$ ) were either lost to follow-up or failed to resolve illness.

At bivariate analysis, death was significantly associated with the following factors: male sex, age $>19$ yrs, pulmonary TB and a previous history of TB (table 2). MDR was associated with death and this association was nearly twice as great in cases with a previous history of TB (secondary MDR, OR 3.1) than in cases without (primary MDR, OR 1.6). The year of notification had no influence on death. The risk of death was significantly higher in nine countries compared with the Netherlands (which was used as the reference). The risk of dying was between two and five times higher in European subjects compared with cases from elsewhere. The association with smear-positive pulmonary TB was not statistically different than for smear-negative cases (data not shown). In multivariate analysis, death remained significantly associated with male sex, increasing age, European origin, pulmonary localisation and MDR, with a risk of death greater for secondary MDR (OR 3.6, 95\% CI 3.0-4.3) than for primary MDR (OR 2.5, 95\% CI 2.0-3.1). Two countries had ORs significantly lower than the reference country, i.e. Portugal and the Czech Republic.

\section{DISCUSSION}

The present analysis was based on TB surveillance data reported by 15 industrialised or fairly well-developed European countries. The data were case-based and pooled over 3 yrs. A number of demographic and clinical factors related to the risk of dying among notified TB cases were identified. The strongest independent risk factors of death were advancing age and MDR, although male sex, European ethnicity, pulmonary TB and country of report also showed significant association. Clinicians and other health professionals should be aware of these findings, which may be relevant to their practice.

A number of limitations are highlighted. When generalising findings, it should be taken into consideration that this analysis was limited to culture-positive patients with available results for drug susceptibility testing. Countries included have had drug-resistance surveillance for a number of years and their laboratories perform well on quality assurance [6]. No outcome data were available for a number of large European countries,

\section{TABLE 1 Observed and expected deaths among tuberculosis (TB) patients in 13 European Union countries ${ }^{\#}$ in 2003}

\begin{tabular}{|c|c|c|c|c|c|}
\hline $\begin{array}{l}\text { Age group } \\
\text { yrs }\end{array}$ & $\begin{array}{c}\text { Total mortality rate } \\
\cdot 1000^{-1}\end{array}$ & TB cases $n$ & Observed deaths $\mathrm{n}(\%)$ & Expected deaths $n$ & $\begin{array}{l}\text { Observed deaths/ } \\
\text { expected deaths }\end{array}$ \\
\hline 0-19 & 0.44 & 2234 & $17(0.8)$ & 0.97 & 17.46 \\
\hline $40-59$ & 3.88 & 8819 & $639(7.2)$ & 34.25 & 18.66 \\
\hline 60+ & 40.20 & 7549 & $1796(23.8)$ & 303.46 & 5.92 \\
\hline
\end{tabular}

\#: Austria, the Czech Republic, Estonia, Germany, Ireland, Latvia, Lithuania, the Netherlands, Portugal, Slovakia, Slovenia, Sweden and the UK; ": from Eurostat [7] and the United Nations Populations Division [8] 
TABLE 2 Profile of tuberculosis (TB) cases according to vital status, and results of bivariate and multivariable analysis in the European Union (EU) during 2002-2004

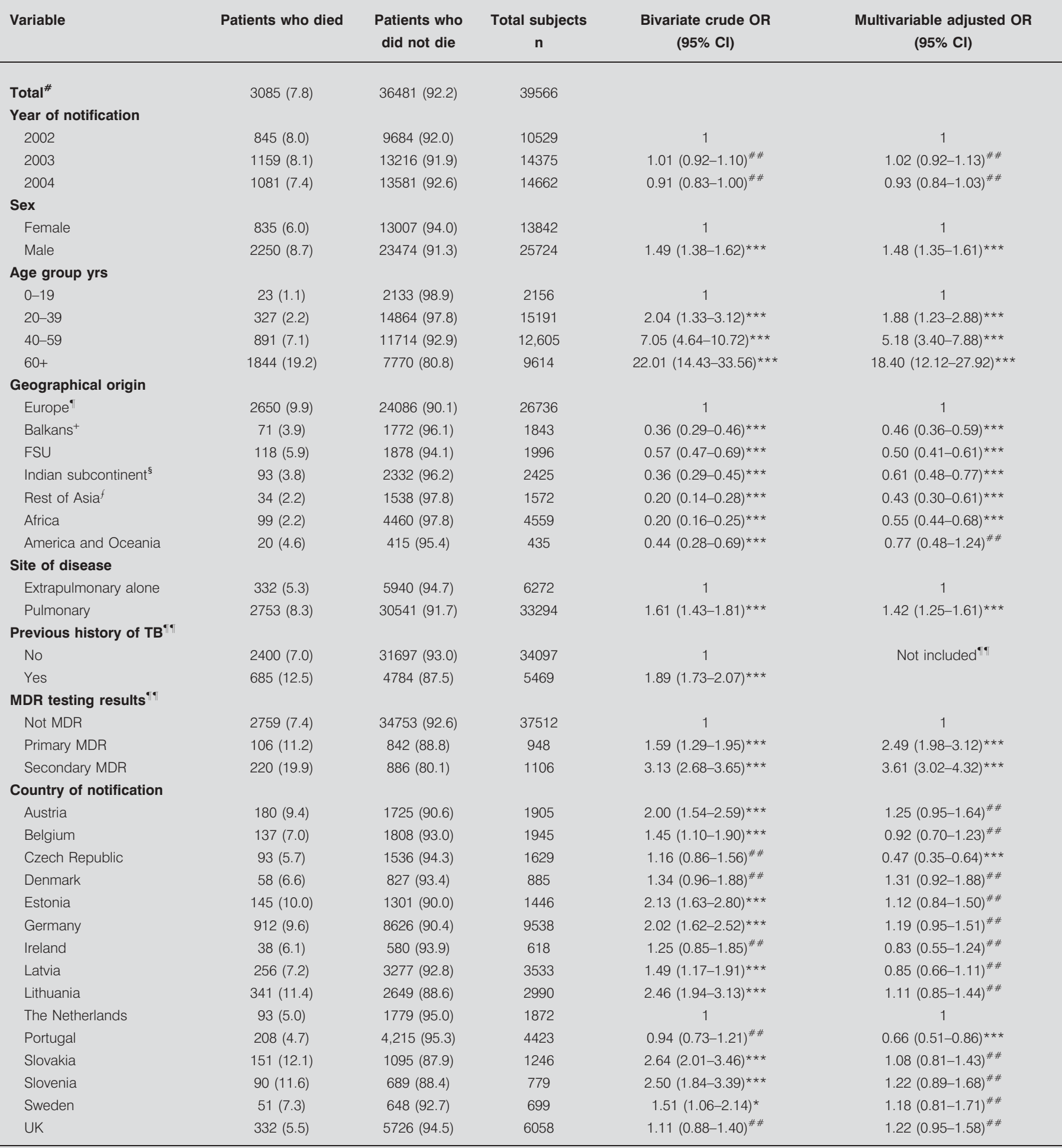

Data are presented as $n(\%)$, unless otherwise indicated. OR: odds ratio; Cl: confidence interval; FSU, Former Soviet Union countries (Armenia, Azerbaijan, Belarus, Georgia, Kazakhstan, Kyrgyzstan, Moldova Republic, Russian Federation, Tajikistan, Turkmenistan, Ukraine, Uzbekistan); MDR: multidrug resistance (resistance to at least isoniazid and rifampicin). ${ }^{*}$ : including all culture-positive cases with results on initial susceptibility testing to isoniazid and rifampicin, and excluding cases with missing data on age, sex, country of origin and site of disease. ". EU and other non-EU countries including Baltic States (Estonia, Latvia, Lithuania); ${ }^{+}$: Albania, Bosnia and

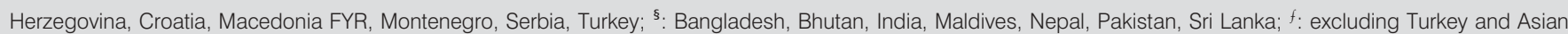
FSU countries; ${ }^{\# \#}$ : nonsignificant; $"$ ": interaction between previous history of TB and MDR was taken into account by recoding MDR status in not MDR, primary MDR (without previous history of TB) and secondary MDR (with previous history). ${ }^{*} p<0.05$; ${ }^{\star \star *}: p<0.001$. 
such as France, Italy and Spain. The immediate cause of death of TB patients was not available. Other studies have shown that this is frequently not directly related to TB [9] and may explain the differences in the likelihood of dying between countries. While $7.8 \%$ of patients died, a substantial proportion of the patients lost to follow-up or still on treatment at 12 months may also have died. Moreover, inclusion of cases detected at post mortem and classification of all cases dying in the outcome category "died", as is recommended, may vary between countries. Intercountry differences may reflect the heterogeneity of patient profiles, as well as different practices in patient recruitment, diagnostic delays, reporting completeness, extent of disease [10] and the specialisation of carers [3]. Nonetheless, the adjusted risk of dying was comparable between most countries, attesting, to a degree, to the validity of pooled analyses of European surveillance data.

Efforts should be made to ensure the completeness of reporting of treatment outcome among TB patients, using information from vital registration to complete reporting of deaths where possible. Information not currently available in the European dataset but which may be relevant to this type of study and to public health action could be collected in future. The contribution of HIV is important [11]. Factors, such as chronic obstructive pulmonary disease, alcohol abuse, drug use, malignancy, diabetes and tobacco smoking, which have also been described, may be relevant $[9,11-13]$. The relationships observed in the present study may partly be explained by the effect of such confounders, the frequency of which is expected to vary according to age and sex. The higher risk in the elderly is partly due to increased comorbidity [4]. The association with male sex could be a consequence of repeated short treatment interruptions among males, as has been documented in different settings; better case-holding may improve outcomes [14].

The association between pulmonary $\mathrm{TB}$ and death is amplified by the rarity of severe forms of extrapulmonary TB in the EU. In 2005, meningitis and disseminated forms of TB represented $<2 \%$ of all TB cases reported [6]. TB patients in the EU originating from high-prevalence countries were less likely to die than European patients. This "healthy migrant effect" has been reported elsewhere [15] and has been attributed to the better physical condition of the average foreign patient when compared with the native. However, it may also be more likely for a migrant worker with $\mathrm{TB}$ to be detected earlier in the course of disease as a result of active screening programmes, with consequent benefits from more timely care [16].

Finally, given the strong risk of dying with multidrug resistance, strict adherence to prescribed treatment regimens, early drug susceptibility testing and the use of adequate medication are crucial to avert the emergence and propagation of drug resistance. This is particularly relevant in the Baltic States and other countries of the former Soviet Union, where multidrug resistance is highly prevalent.

\section{ACKNOWLEDGEMENTS}

The authors would like to thank J-C. Desenclos (Dept of Infectious Diseases, Institut de Veille Sanitaire, Saint-Maurice, France) for his useful contribution to the interpretation of the data. The authors also thank the following EuroTB national contacts who supplied the data and who were invited to comment on an early draft of this article: J.P. Klein (Federal Ministry for Health and Women, Vienna, Austria); M. Wanlin (Belgium Lung and Tuberculosis Association, Brussels, Belgium); J. Wallenfels (National Tuberculosis Surveillance Unit, Prague, Czech Republic); P. Andersen (Statens Serum Institute, Copenhagen, Denmark); V. Hollo (National TB Register, Tallinn, Estonia); W. Haas and B. Brodhun (Robert Koch Institut, Berlin, Germany); J. O'Donnell (HSE Health Protection Surveillance Centre, Dublin, Ireland); J. Leimans (State Centre of Tuberculosis and Lung Diseases, Riga, Latvia); E. Davidaviciené (Centre of Lung Diseases and Tuberculosis, Vilnius, Lithuania); C. Erkens (KNCV Tuberculosis Foundation, The Hague, the Netherlands); A. Fonseca Antunes (Directorate General of Health, Lisbon, Portugal); I. Solovic (Institute for TB, Respiratory Diseases and Thoracic Surgery, Vysné Hagy, Slovakia); D. Erzen (University Clinic of Respiratory and Allergic Diseases, Golnik, Slovenia); V. Romanus (Institute for Infectious Disease Control, Solna, Sweden); and for the UK, J. McMenamin (Health Protection Scotland, Glasgow), R. Salmon (CDSC Wales, Cardiff), B. Smyth (Communicable Disease Surveillance Centre, Belfast) and J. Watson (Health Protection Agency, London). Comments were also invited from other experts sitting on the EuroTB Advisory Committee, including the following: L. Clancy (UNION, Paris, France), F. Drobniewski (HPA National Mycobacterium Reference Unit, London, UK), E. Ibraim (Pneumology Institute "Marius Nasta", Bucharest, Romania), M. Forssbohm (Landeshauptstadt, Wiesbaden, Germany), M. KorzeniewskaKosela (National Institute of Tuberculosis and Lung Diseases, Warsaw, Poland), P. Ruutu (KTL National Public Health Institute, Helsinki, Finland), V. Kuyvenhoven (KNCV Tuberculosis Foundation, The Hague, the Netherlands) and R. Zaleskis (WHO/Euro, Copenhagen, Denmark). A special tribute is due to A. Infuso, who coordinated the EuroTB network from 2000 until his death in office in 2005.

\section{REFERENCES}

1 World Health Organization. Global tuberculosis control: surveillance, planning, financing. WHO report 2007. (WHO/HTM/TB/2007.376). Geneva, WHO, 2007.

2 van Lier E, Havelaar A, Nanda A. The burden of infectious diseases in Europe: a pilot study. Euro Surveill 2007; 12: E3-E4.

3 Vasankari T, Holmstrom P, Ollgren J, Liippo K, Kokki M, Ruutu P. Risk factors for poor tuberculosis treatment outcome in Finland: a cohort study. BMC Public Health 2007; 7: 291.

4 Zevallos M, Justman JE. Tuberculosis in the elderly. Clin Geriatr Med 2003; 19: 121-138.

5 EuroTB. Surveillance of tuberculosis in Europe. WHO Collaborating Centre. www.eurotb.org Date last updated: February 14, 2008. Date last accessed: December 1, 2007.

6 EuroTB and the national coordinators for tuberculosis surveillance in the WHO European Region. Surveillance of tuberculosis in Europe. Report on tuberculosis cases notified in 2005. Saint-Maurice, Institut de Veille Sanitaire, 2007.

7 European Commission Eurostat, www.epp.eurostat.ec. europa.eu Date last accessed: December 17, 2007. 
8 United Nations Population Division. Annual Population 1950-2050 (The 2006 revision). New York, United Nations, 2007.

9 Sterling TR, Zhao Z, Khan A, et al. Mortality in a large tuberculosis treatment trial: modifiable and nonmodifiable risk factors. Int J Tuberc Lung Dis 2006; 10: 542-549.

10 Walpola HC, Siskind V, Patel AM, Konstantinos A, Derhy P. Tuberculosis-related deaths in Queensland, Australia, 1989-1998: characteristics and risk factors. Int J Tuberc Lung Dis 2003; 7: 742-750.

11 Zumla A, Malon P, Henderson J, Grange JM. Impact of HIV infection on tuberculosis. Postgrad Med J 2000; 76: 259-268.

12 Alisjahbana B, Sahiratmadja E, Nelwan EJ, et al. The effect of type 2 diabetes mellitus on the presentation and treatment response of pulmonary tuberculosis. Clin Infect Dis 2007; 45: 428-435.

13 Bates MN, Khalakdina A, Pai M, Chang L, Lessa F, Smith KR. Risk of tuberculosis from exposure to tobacco smoke: a systematic review and meta-analysis. Arch Intern Med 2007; 167: 335-342.

14 Balasubramanian R, Garg R, Santha T, et al. Gender disparities in tuberculosis: report from a rural DOTS programme in south India. Int J Tuberc Lung Dis 2004; 8: 323-332.

15 Borgdorff MW, Veen J, Kalisvaart NA, Nagelkerke N. Mortality among tuberculosis patients in The Netherlands in the period 1993-1995. Eur Respir J 1998; 11: 816-820.

16 Laifer G, Widmer AF, Simcock M, et al. TB in a lowincidence country: differences between new immigrants, foreign-born residents and native residents. Am J Med 2007; 120: 350-356. 